\title{
Earth's Atmospheric Electricity Parameter Response During Venus Transit
}

\author{
Syam Sundar De *, Suman Paul, Sudarsan Barui, Dilip Kumar Haldar, and Gautam Guha \\ Centre of Advanced Study in Radio Physics and Electronics, University of Calcutta, Kolkata, India
}

Received 29 January 2014, revised 6 September 2014, accepted 28 October 2014

\begin{abstract}
Venus transited across the Sun on 06 June 2012, introducing significant contribution to the tidal characteristics of the solar atmosphere. Earth's atmosphere was perturbed due to an anomalous Coronal Mass Ejection (CME) and $\gamma$-radiation influenced by the solar tide due to Venus transit, thereby the Earth-ionosphere waveguide characteristics were changed. In this anomalous situation we measured some atmospheric electricity parameters such as Schumann resonance (SR) amplitude, very low frequency (VLF) sferics, subionospheric transmitted signals and the point discharge current (PDC) along with the vertical electrical potential gradient (PG) at the ground surface on the day of transit. The results showed some remarkable variations during the transit as well as pre- and post-transit periods. The observed anomalies in the recorded data were interpreted in terms of the anomalous solar tidal effects initiated due to Venus transit.
\end{abstract}

Key words: Venus transit, Coronal Mass Ejection (CME), Earth-ionosphere waveguide

Citation: De, S. S., S. Paul, S. Barui, D. K. Haldar, and G. Guha, 2015: Earth's atmospheric electricity parameter response during Venus transit. Terr. Atmos. Ocean. Sci., 26, 135-143, doi: 10.3319/TAO.2014.10.28.01(AA)

\section{INTRODUCTION}

The physical behaviour and properties of the upper atmosphere are fully dependent on the incoming solar radiations constituting ultraviolet (UV), $\mathrm{X}-, \boldsymbol{\gamma}$-rays etc.. Different atmospheric electricity properties such as very low frequency (VLF) sferics, atmospheric potential gradient (PG), air-earth current, Schumann resonance (SR) are directly and indirectly connected with the influence of solar radiation along with the outcome of some geophysical events. The parameters showed fluctuations under different situations (Kumar and Singh 2013).

Among those situations, SR phenomena within the Earth-ionosphere waveguide occur continuously due to constructive natural electromagnetic wave interference in the extremely low frequency $(\mathrm{ELF})$ band $(8,14,20,26, \ldots \mathrm{Hz})$, where $8 \mathrm{~Hz}$ is the $1^{\text {st }}$ mode representing the wave having a wavelength equal to the circumference of the Earth $(\sim 40000 \mathrm{~km})$, interfering with the $2 \pi$ phase difference. These electromagnetic radiations, originating from lightning discharges, are trapped in the Earth-ionosphere cavity. These waves circulate several times around the spherical waveguide before dissipation (Sentman 1995). SR stipulates a global monitor-

\footnotetext{
* Corresponding author

E-mail:de_syam_sundar@yahoo.co.in
}

ing process of the lower ionospheric condition.

Another geophysical event, the sferics are observed in near equatorial zones, spread out along the Earth-ionosphere waveguide and are helpful in the prediction of storms, cyclones, tsunami and other irregular complicated weather phenomena (Molinari et al. 1999; Lay et al. 2004; Rodger et al. 2006).

The variations in SR intensity, frequency, Q-values, air-temperature, electric field, air-earth current, and conductivity over the Earth's surface at tropical latitudes $\left( \pm 25^{\circ}\right)$ and temperate latitudes $\left( \pm 60^{\circ}\right)$ during fair-weather and under disturbed conditions are interrelated with the solar radiations, global thunderstorm activity as well as concentration and nature of aerosol content in the lower atmosphere (Williams 1994; Tonev and Velinov 1996; Bister 2001; De et al. 2006a, 2008; Sorokin et al. 2006).

Coronal Mass Ejection (CME) is a continuous phenomena occurring from the Sun's entire coronal zone responsible for the outflow of solar masses, e.g., protons, electrons, neutrons, and solar wind in the form of plasma (Gopalswamy and Thompson 2000; Webb 2000; Li et al. 2003; Jacobs and Poedts 2011). The solar wind penetrates into the Earth's atmosphere through the magnetopause. The solar atmosphere consists of very high temperature plasma 
and is fully dynamic in nature. Due to its high dynamic activity, huge magnetic dipoles are continuously generated with intense magnetic fields.

The energetic charged particles released from the solar corona are trapped within the dipole field. Those particles will gyrate along the magnetic field lines and accumulate as sun spots. These field lines are gradually elongated outwards from the Sun due to the radial momentum of the massive charged particles trapped in the field ultimately causing breakdown in the field lines at their critical limit, thus establishing magnetic reconnection with the interplanetary magnetic field. This process evolves into huge energy as electromagnetic radiation and releases the trapped masses along with the electromagnetic radiation in the form of solar X-rays and $\gamma$-ray bursts (Masuda et al. 1994; Shibata 1996; Kahler and Reames 2003; Kasatkina et al. 2009; Murphy et al. 2012). This intense radiation is responsible for ionizing the constituent particles of the D-region of the ionosphere. The D-region is thereby highly modified and thus, the characteristic behaviour of the Earth-ionosphere waveguide is abruptly changed.

In the area of atmospheric electricity we are taking continuous SR, PG, point discharge current (PDC), and VLF sub-ionospheric transmitted signal records at $19.8 \mathrm{kHz}$ and also VLF sferics at frequencies of 7 and $9 \mathrm{kHz}$ over Kolkata (Lat. $22.56^{\circ} \mathrm{N}$, Long. $88.5^{\circ} \mathrm{E}$ ) to detect and interpret the effects of different solar and geophysical events. The Venus transit effects on FWF (Fair Weather Field), ELF-VLF amplitudes, atmospheric vertical PG, different modes of SR on 8 June 2004 were investigated from this centre and reported (De et al. 2006b). The unusual variation in SR signals, VLF sferics, VLF transmitted subionospheric signal, PG and PDC from the Earth's surface at Kolkata corroborated the Venus transit on 06 June 2012. The influences of CMEs and $\gamma$-ray bursts from the solar corona in the solar tidal effect process were taken as the key process in the observed Venus transit phenomenon.

\section{EXPERIMENTAL ARRANGEMENT}

\subsection{SR}

The SR parameters have been recorded regularly for the last several years at Kolkata using square loop antenna. Two such loops were mounted on a wooden structure connected in series and their effective gain was increased beyond $5 \mathrm{~dB}$ with this arrangement. One square loop has $1 \mathrm{~m}$ length on each side with a total of 75 turns. The other square loop has 90 turns and $1.3 \mathrm{~m}$ length on each side. A co-axial cable $30 \mathrm{~m}$ in length is used to transfer the signal from the antenna into the receiver input where it is pre-amplified. A stereo-preamplifier integrated circuit with LA3161 chip was chosen with low frequency response starting below $5 \mathrm{~Hz}$ and extending up to a few $\mathrm{kHz}$. This arrangement handles input voltage ranging from few $\mu \mathrm{V}$ to $\mathrm{mV}$. The frequency selective stages were designed with active circuit elements. A wide-band amplifier with the output taken through an active low-pass filter with a cut-off frequency near $35 \mathrm{~Hz}$ further amplifies the signal. This signal information is now stored in a computer through a Data Acquisition System (DAS) card in which an $80 \mathrm{~Hz}$ generator is used to convert the analog signal into digital satisfying the Nyquist criterion. A wide-band very low input voltage sensitive and low frequency sensitive receiver was designed to detect the input signal. The square loop antenna has a very narrow bandwidth sensitivity at $5-50 \mathrm{~Hz}$. This system is not sensitive to the VLF range.

\subsection{VLF Sferics and VLF Transmitted Sub-Ionospheric Signals}

VLF spheric signals at frequencies of 7 and $9 \mathrm{kHz}$ and transmitted signals at $19.8 \mathrm{kHz}$ were regularly recorded for the last several years from Kolkata. A straight horizontal copper wire 8 SWG $120 \mathrm{~m}$ in length is used in the form of an inverted $\mathrm{L}$ type antenna. This antenna, installed $10 \mathrm{~m}$ above the ground is capable of receiving vertically polarized transmitted signals in the VLF bands from different transmitting stations. These signals are recorded through a PCI 1050, 16 channel 12 bit DAS card and then processed and stored in a computer. The receiver bandwidth used for the 7 and $9 \mathrm{kHz}$ signals is set to $100 \mathrm{~Hz}$ to make the measurement sensitive to modal interference patterns (De et al. 2013a).

\subsection{PG and PDC}

The vertical electric field has been measured on a continuous basis with an ac field-mill. The alternating signal from the field-mill is amplified using a signal processor with one-second time constant. IC LF356N has been used at the amplifier input stage because of its high input resistance $\left(\sim 10^{12} \Omega\right)$ and good signal to noise ratio. The field-mill sensitivity has been calculated as $(0.33 \pm 0.03) \mathrm{Vm}^{-1}$. The output is recorded using the same DAS card at a sample rate of one data per second.

The PDC continuous measurement is made using a sharp point of a wire tip situated about $8 \mathrm{~m}$ above the ground. The transient responses from the wire tip are amplified. The amplifier output is recorded in a computer. The r.m.s. value of the filtered data are analysed regularly using Origin 5.0 software (De et al. 2013b).

\section{OBSERVATIONAL RESULTS AND DISCUSSIONS}

The temporal variations in the amplitude of the first three SR modes on 06 June 2012, during the Venus transit are shown in Fig. 1, together with their averaged values for 24 control days: 12 days before the event and 12 days after event 
commencement, i.e., the average of the data obtained from 25 May 2012 to 18 June 2012 except for 06 June 2012. Standard deviations from the average values were plotted as error bars in the average plot. The average amplitude of the first mode is about $0.15 \mathrm{AU}$ (Arbitrary Unit), but a gradual increase with highest values at about $0.27 \mathrm{AU}$ at $09 \mathrm{~h}$ local time, whereas on the day of transit, the corresponding amplitude decreases gradually from the local time $00-05 \mathrm{~h}$ from $0.21-0.12 \mathrm{AU}$. After that the amplitude remains steady with a small incremental value at $09 \mathrm{~h}$ local time. After $12 \mathrm{~h}$ its value increases. Data were chosen in arbitrary units because we require the relative change, for which we did not use measurement data calibration to avoid the experimental determination of several antenna constants.

The second mode amplitude in the average value graph remains almost constant (about $0.16 \mathrm{AU}$ ) up to $08 \mathrm{~h}$ local time with a small decremented value of about $0.11 \mathrm{AU}$ at $05 \mathrm{~h}$ and then increases up to its highest value of about $0.24 \mathrm{AU}$ at
$09 \mathrm{~h}$ local time. After that the average amplitude decreases. On the day of transit the corresponding amplitude shows a gradual decrease in values from $0.18-0.08 \mathrm{AU}$ at $08 \mathrm{~h}$ local time which then gradually increases.

The $3^{\text {rd }}$ mode amplitude in the average value graph shows a gradual decrease from likely $0.135-0.10 \mathrm{AU}$ at $04 \mathrm{~h}$ local time and then increases and remains constant. After $12 \mathrm{~h}$ its value again increases. On the day of transit the corresponding amplitude decreases from about $0.15-0.11 \mathrm{AU}$ at $05 \mathrm{~h}$ local time and then increases up to its maximum value of about $0.15 \mathrm{AU}$ at $09 \mathrm{~h}$ local time followed by a gradual decrease of about $0.09 \mathrm{AU}$ at $12 \mathrm{~h}$ local time. After that the corresponding amplitude increases.

The cumulative impact of CME and solar X-rays and $\gamma$-ray bursts on the Earth's atmosphere, D-region fluctuations in height and irregular variations in medium characteristics may be responsible for the disparity in the nature of the variation in $3^{\text {rd }}$ mode relative to the $1^{\text {st }}$ and $2^{\text {nd }}$ modes.
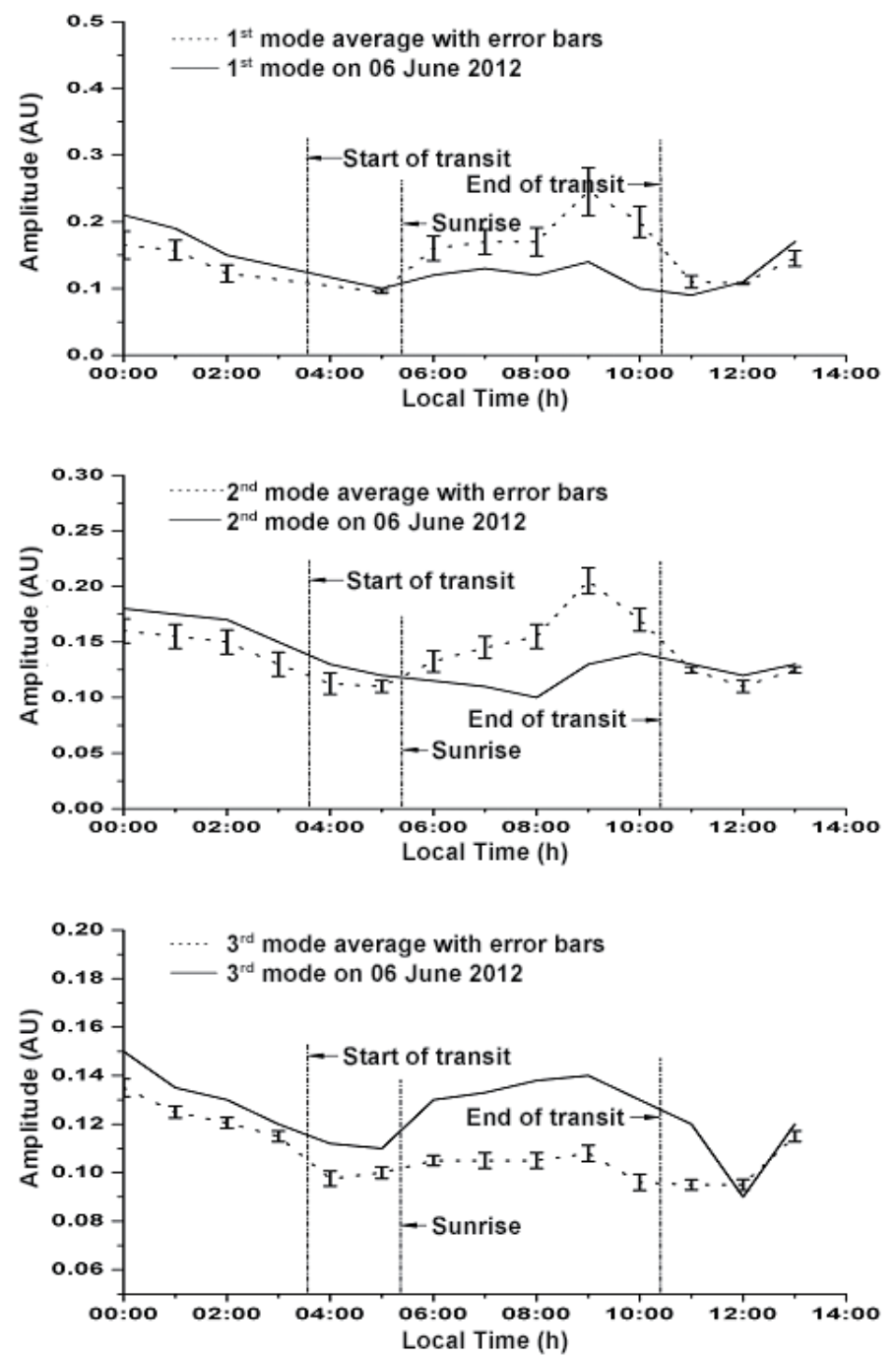

Fig. 1. Temporal variations of the amplitude of the first three Schumann resonance (SR) modes on 06 June 2012, together with their averaged values of 24 control days. Standard deviations from the average values have been plotted as error bars in the average plot. 
As the Earth-ionosphere waveguide is influenced during the Venus transit, there should also be some frequency changes SR modes because of conductivity variations in the medium. The height variation of the D-region is also responsible for the modal frequency variation. Unfortunately we missed the opportunity to record those activities.

The sferics generated mainly from the lightning discharges propagates through the Earth-ionosphere waveguide throughout the globe maintaining an ambient value in its amplitude. The magnitude of the received signals affects the $9 \mathrm{kHz}$ signal more because the lightning intensity generated electromagnetic waves are dominated surrounding $9-10 \mathrm{kHz}$ (De et al. 2011). That is why we choose $9 \mathrm{kHz}$ and a surrounding available frequency $7 \mathrm{kHz}$ in this study. Comparative plots of the Integrated Field Intensity of Sferics (IFIS) at $9 \mathrm{kHz}$ on 06 June 2012, during the Venus transit (continuous line) and its 24 days average value over the same period (dotted line) are depicted in Fig. 2. On the day of transit some significant peaks were observed just before and after the start of the transit. The IFIS amplitude then slowly increased to a steady value showing remarkable fluctuations before and after the end of the transit.

Figure 3 also shows a comparative plot of the IFIS at $7 \mathrm{kHz}$ on 06 June 2012, during the Venus transit (continuous line) and its 24 days average value over the same period (dotted line). On the Venus transit day the IFIS amplitude showed a steady value just before and after the start of the transit. The corresponding amplitude increased gradually to a steady value showing some significant fluctuations just before the end of the transit. After the transit the IFIS amplitude fluctuated to a small extent.

The Venus transit day looks different from the average well before and well after the transit. At the start of the transit the position of the Sun, Venus, and Earth were nearly in the same line, so the enhancement of all kinds of tidal effects for the pre-transit as well as post-transit signatures may be possible. Although towards the end of transit the deviation was small, throughout the day the signal variation was quite remarkable. The amplitude deviation from the error bar indicated that the day was quite unusual. Due to the transit the D-region was highly disturbed as stated, so the reflection layer of the lower ionosphere was highly irregular and multiple reflections were also irregular. The attenuation of the sferics signal within the Earth-ionosphere waveguide was therefore greater. This may be the possible reason for the observed decrease.

A comparative picture of the VLF sub-ionospheric transmitted signal at $19.8 \mathrm{kHz}$, transmitted from North-West Cape, Australia (Lat. $21.82^{\circ} \mathrm{S}$, Long. $114.16^{\circ} \mathrm{E}$ ) is presented in Fig. 4 from the 06 June 2012 record during the Venus transit (continuous line) and its 24 day average value over the same period (dotted line). This signal was operated continuously at $1000 \mathrm{~kW}$ radiated power, transmitted from an omni-directional antenna. The transmitting station is $5665 \mathrm{~km}$ away from our Kolkata receiving station. On the day of transit a regular fluctuation was found just before and after the beginning of the transit with a deep decrement occurring during the sunrise period. The transmitted signal amplitude then slowly increased to its steady value showing a fluctuation before and after the end of the transit. The lower ionosphere is continuously under the effects of the CME, with solar flares and bursts before and after the transit.

The temporal variation in the vertical PG on 06 June 2012 during the Venus transit (continuous line) and its 24 day average value over the same period (dotted line) are presented in Fig. 5 with standard deviations from the average value plotted as error bars. On the day of transit the maximum and minimum PG values were 230 and $140 \mathrm{Vm}^{-1}$ respectively. During other days it was around 180 and $160 \mathrm{Vm}^{-1}$, respectively. Around the mid-transit period, i.e., $08 \mathrm{~h}$ local time, the PG value attained its maximum value of $230 \mathrm{Vm}^{-1}$. Higher levels of pollution at the place of recording may be responsible for this variation. The variation in PG is well beyond the standard deviation for the average trend. Thunderstorms strongly impact the variation in PG. There were no thunderstorm and lightning occurrences throughout the day of transit in the nearby area of our receiving station and no significant effects were observed.

The temporal variation in PDC is plotted in Fig. 6 from the recorded data of 06 June 2012. It is shown by the continuous line curve and the 24-day average over the same period is shown by the dotted line. The standard deviations from the average value are plotted as error bars in the figure. After the start of the transit the PDC value increased gradually and attained its maximum value $2.59 \times 10^{-10} \mathrm{Am}^{-2}$ at $10 \mathrm{~h}$ local time and then gradually decreased. During the same period, on other days, its maximum and minimum values were $2.48 \times 10^{-10}$ and $2.32 \times 10^{-10} \mathrm{Am}^{-2}$, respectively. On the day of transit the minimum value showed $2.27 \times 10^{-10} \mathrm{Am}^{-2}$. The deviation from the average starts well before the transit begins, flips polarity in the middle of the transit and then extends beyond the end of the transit. During the period of transit the effects of CMEs and $\gamma$-rays are highest upon the Earth's atmosphere causing enhanced ionization process (Brajša et al. 2009). As a result the PDC starts increasing and then gradually falls after the transit. The polarity flips in the middle of the transit due to the combined effects of the external sources upon the atmosphere. Quite a long time before the Venus transit, the tidal effects remained operative, which flips the polarity and continues for a longer time until the influence ceased. The local increment of aerosol concentration may also be responsible for this type of variation on the day of transit.

During the transit period both the two atmospheric electricity parameters gradually increased after the start of transit. The local temperature did not show any remarkable change during the transit. The relative humidity remained a little higher than its normal value throughout the day. 


\section{CONCLUSIONS}

On the day Venus transited across the sun (06 June 2012) experiments were conducted at Kolkata to observe the effects, if any, of the Venus transit on FWF, ELF-VLF amplitudes, different SR modes, atmospheric vertical PG and PDC.

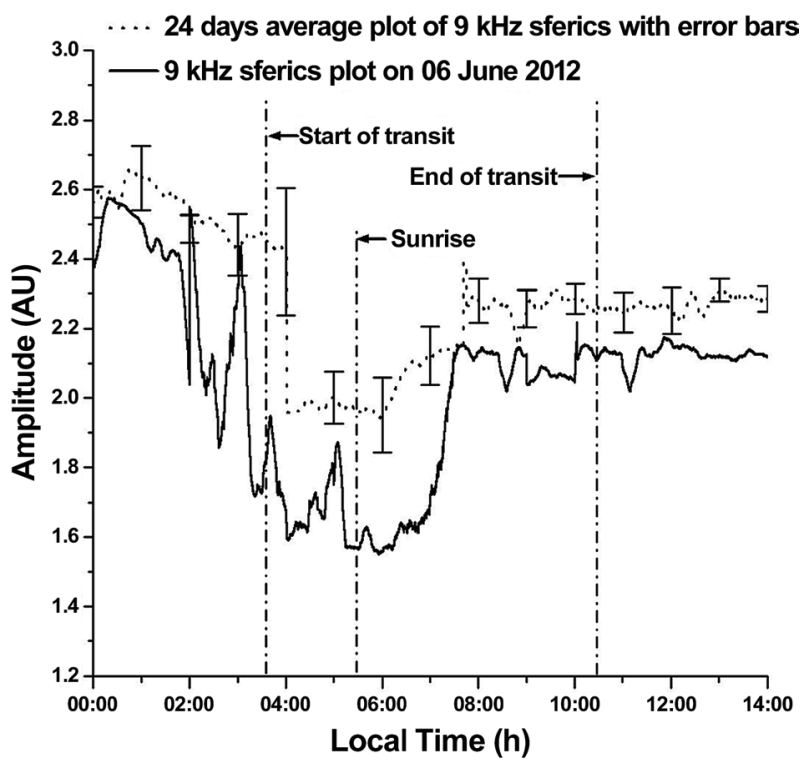

Fig. 2. Variations of Integrated Field Intensity of Sferics (IFIS) at $9 \mathrm{kHz}$ on 06 June 2012 (continuous line) and its 24 control days average value over the same period (dotted line). Standard deviations from the average value are plotted as error bars.

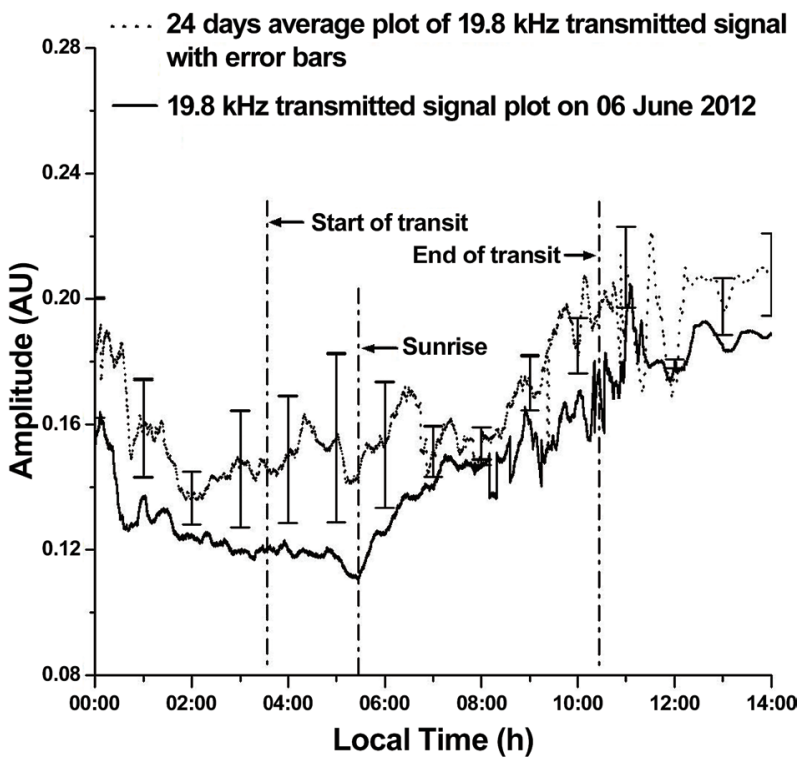

Fig. 4. Variations of very low frequency (VLF) sub-ionospheric transmitted signal at $19.8 \mathrm{kHz}$, transmitted from North west cape, Australia (Lat. $21.82^{\circ} \mathrm{S}$, Long. $114.16^{\circ} \mathrm{E}$ ) on 06 June 2012 (continuous line) and its 24 control days average value over the same period (dotted line). Standard deviations from the average value are plotted as error bars.
The atmospheric variation parameter trend was similar.

To ascertain whether the magnetic activity has any significant role we checked the diurnal variation in the Dst and $\mathrm{Kp}$ index for May and June 2012 available from http:// wdc.kugi.kyoto-u.ac.jp/wdc/Sec3.html which are presented in Figs. 7 and 8 respectively. The Dst index is a measure of

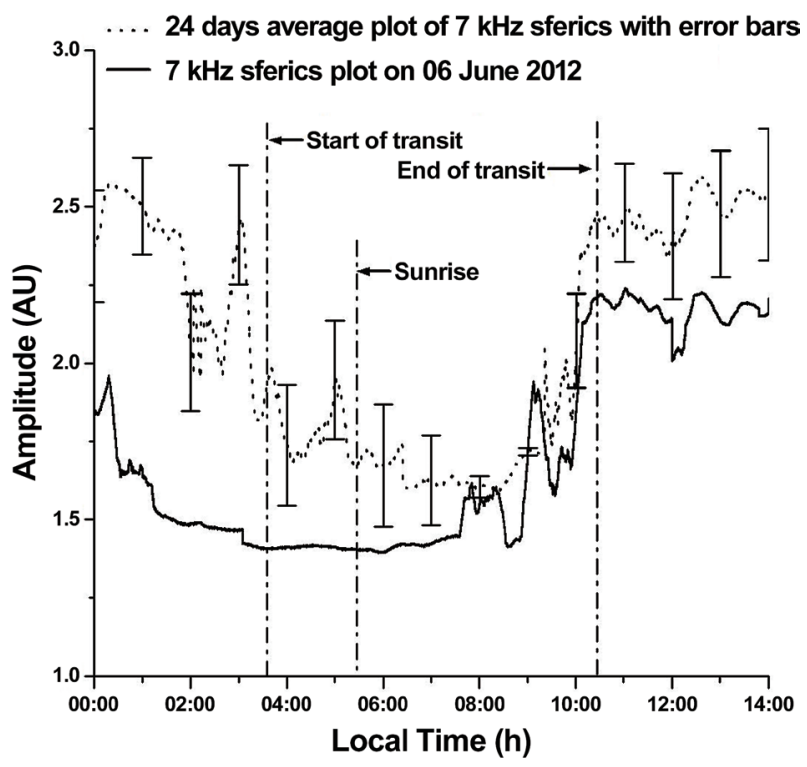

Fig. 3. Variations of Integrated Field Intensity of Sferics (IFIS) at $7 \mathrm{kHz}$ on 06 June 2012 (continuous line) and its 24 control days average value over the same period (dotted line). Standard deviations from the average value are plotted as error bars.

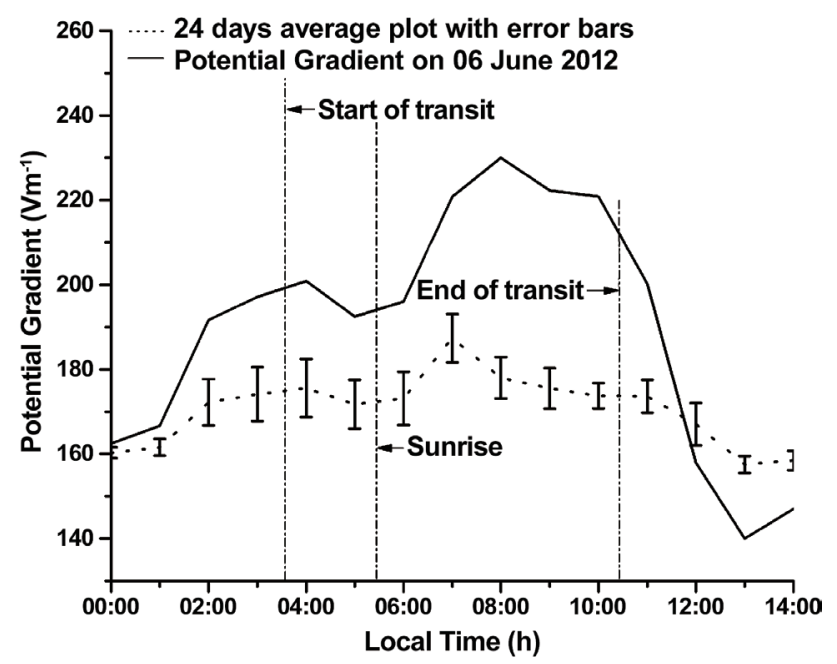

Fig. 5. Temporal variation of vertical potential gradient (PG) on 06 June 2012, during the Venus transit (continuous line) and its 24 control days average value over the same period (dotted line) with standard deviations from the average value plotted as error bars. 


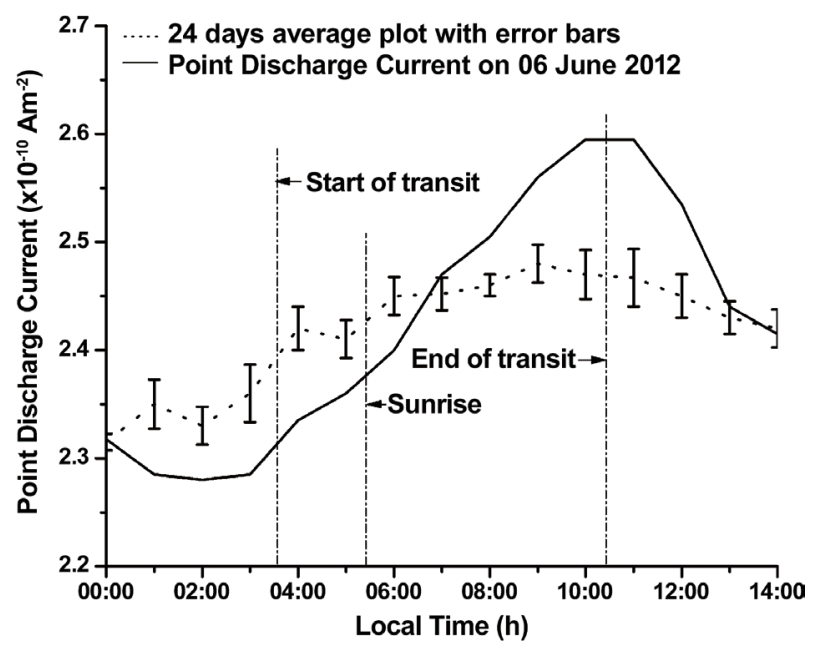

Fig. 6. Temporal variation of point discharge current (PDC) on 06 June 2012 (continuous line) and its 24 control days average value over the same period (dotted line) with standard deviation from the average value plotted as error bars.
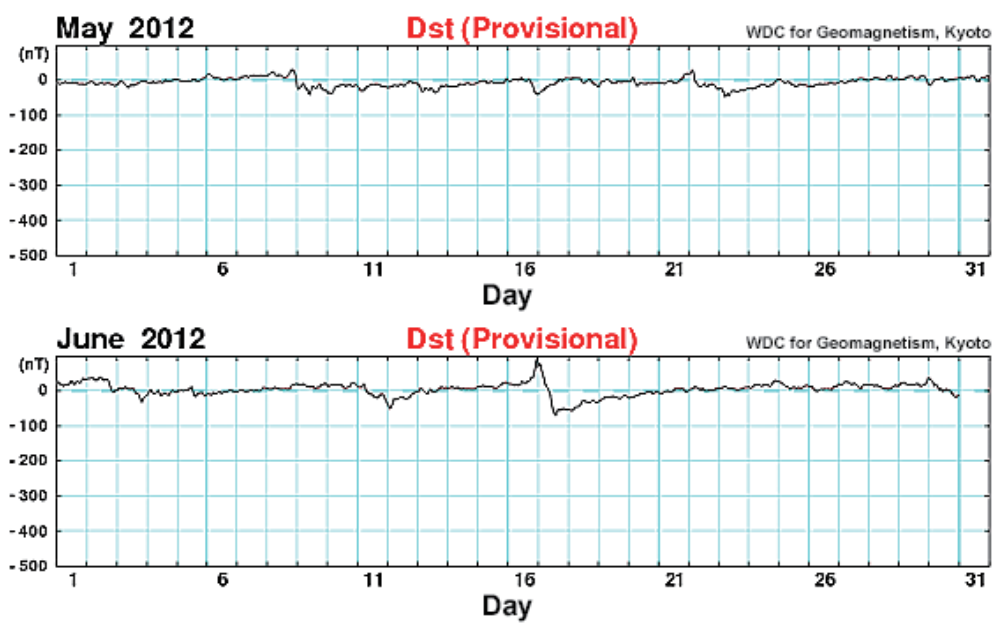

Fig. 7. Plots of Dst values for the month of May and June 2012 except the day of 06 June 2012, the day of Venus transit.

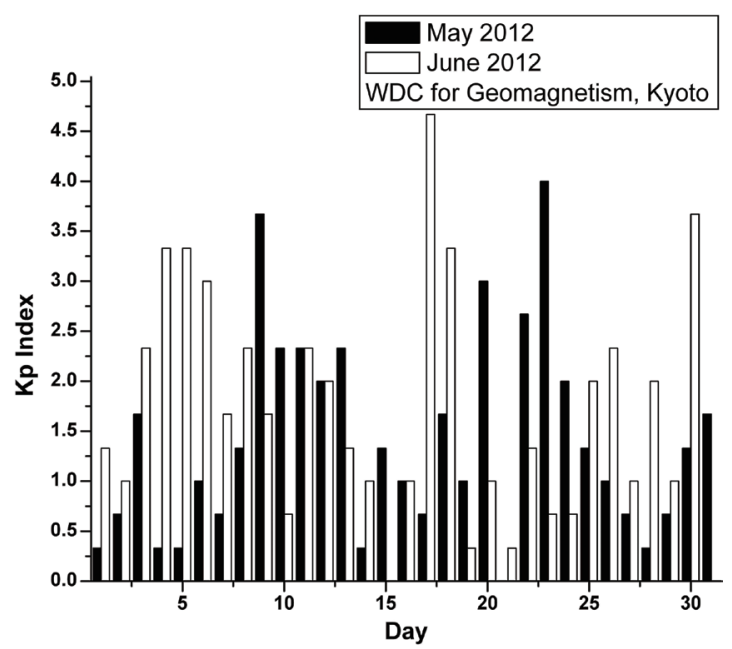

Fig. 8. Plots of Kp index averaged over $9 \mathrm{~h}$ interval within which the complete duration of transit of Venus occurred for the month of May and June 2012. Black bar diagrams are for May while White is for June. 
the variations in geomagnetic field due to the equatorial ring current. At a given time the Dst index is the average of the variations in H-component over all longitudes. The reference level is selected such that Dst is statistically zero on internationally designated quiet days. An index smaller than $-50 \mathrm{nT}$ indicates the absence of magnetic disturbances. It is seen from Fig. 7 that the Venus transit day was a quiet day. Sudden sub-storm commencement (SSC) was observed in the main phase of a geomagnetic storm on 16 and 17 June 2012 , but those had no significant effects on the 24 control day average values. Because the other 22 days showed normal quiet day signatures and June 16 and 17 exhibited opposite impact, which minimized the effect to some extent. The Kp index ranges between $0-9$. The values for this index give a good indication of the geomagnetic activity, whereby values between 0 - 4 indicate quiet magnetic conditions. Figure 8 is drawn for the whole month of May and June 2012 with the Kp index data averaged over the duration within which the Venus transit took place on 06 June 2012. The average Kp index value on the Venus transit day, being 3 , implies that the day was geomagnetically quiet. All these values confirm that the atmospheric electricity parameters observation on the transit day was devoid of any real event such as magnetic disturbances or solar proton events, etc..

All of the planets revolving round the Sun produce gravitational attraction under the central force field producing tidal effects on the solar atmosphere (Hung 2007; Martin 2009). During the tide CME will increase. The gravitational effects from Venus and Earth upon the solar tide are greater than that from the other planets. The Earth, Venus and the Sun come into alignment during the Venus transit. The combined gravitational tidal effects on the solar atmosphere will therefore be very high. Mass ejection from the Sun will also attain maximum. In this way the waveguide characteristics become more anomalous to producing perturbations. As a result of tidal impact solar X-and $\gamma$-rays that perturb the ionospheric D-region will increase, as a result, the Earth's ionosphere waveguide characteristics will change simultaneously and thereby perturb the transmitted VLF and SR signals (Kahler and Reames 2003; Raymond et al. 2003; Kasatkina et al. 2009).

The observations presented in this paper were explained based on the Earth-ionosphere waveguide medium characteristic changes, medium conductivity changes, Dregion height fluctuation, $\mathrm{X}$ - and $\gamma$-ray perturbations caused by CME effects initiated and continued by the Venus transit on 06 June 2012.

Acknowledgements The authors gratefully acknowledge the support of the Indian Space Research Organization (ISRO) through S. K. Mitra Centre for Research in Space Environment, Institute of Radio Physics and Electronics, University of Calcutta, Kolkata 700 009, India in carrying out this work. We are also thankful to the learned Anony- mous Reviewers of this paper for their valuable comments and suggestions, the inclusion of which sufficiently improved this revised version.

\section{REFERENCES}

Bister, M., 2001: Effect of peripheral convection on tropical cyclone formation. J. Atmos. Sci., 58, 3463-3476, doi: 10.1175/1520-0469(2001)058<3463:EOPCOT>2.0.C O;2. [Link]

Brajša, R., H. Wöhl, A. Hanslmeier, G. Verbanac, D. Ruždjak, E. Cliver, L. Svalgaard, and M. Roth, 2009: On solar cycle predictions and reconstructions. Astron. Astrophys., 496, 855-861, doi: 10.1051/00046361:200810862. [Link]

De, S. S., B. K. De, S. K. Adhikari, S. K. Sarkar, R. Bera, A. Guha, and P. K. Mandal, 2006a: A report on some specific features of the atmospheric electric potential gradient in Kolkata. Indian J. Phys., 80, 167-172.

De, S. S., B. K. De, S. K. Adhikari, B. K. Sarkar, S. K. Sarkar, A. Guha, P. K. Mandal, S. K. Mandal, H. P. Sardar, and M. Ray, 2006b: The effect of recent Venus transit on Earth's atmosphere. Ann. Geophys., 49, 1209-1214, doi: 10.4401/ag-3140. [Link]

De, S. S., B. Bandyopadhyay, B. K. De, S. Paul, D. K. Haldar, S. Barui, and S. Bhattacharya, 2008: On some observations of solar and terrestrial phenomena by subionospheric transmitted signals. Bulg. J. Phys., 35, 223-234.

De, S. S., B. Bandyopadhyay, S. Paul, D. K. Haldar, M. Sanfui, B. K. De, S. Chattopadhyay, A. K. Kundu, and S. Barui, 2011: AILA-2009: Its effects on VLF IFIA and probable scientific explanation. Bulg. J. Phys., 38, 433-447.

De, S. S., S. Paul, S. Barui, P. Pal, B. Bandyopadhyay, D. Kala, and A. Ghosh, 2013a: Studies on the seasonal variation of atmospheric electricity parameters at a tropical station in Kolkata, India. J. Atmos. Sol.-Terr. Phys., 105-106, 135-141, doi: 10.1016/j. jastp.2013.09.006. [Link]

De, S. S., S. Paul, S. Barui, P. Hazra, D. Kala, D. K. Haldar, A. Ghosh, and G. Guha, 2013b: Point discharge current during a solar eclipse. Earth Moon Planets, 111, 79-87, doi: 10.1007/s11038-013-9424-4. [Link]

Gopalswamy, N. and B. J. Thompson, 2000: Early life of coronal mass ejections. J.Atmos.Sol.-Terr. Phys., 62, 14571469, doi: 10.1016/S1364-6826(00)00079-1. [Link]

Hung, C. C., 2007: Apparent relations between solar activity and solar tides caused by the planets. Technical Memorandum, NASA Glenn Research Center; Cleveland, OH, United States, $34 \mathrm{pp}$.

Jacobs, C. and S. Poedts, 2011: Models for coronal mass ejections. J. Atmos. Sol.-Terr. Phys., 73, 1148-1155, doi: 10.1016/j.jastp.2010.12.002. [Link] 
Kahler, S. W. and D. V. Reames, 2003: Solar energetic particle production by coronal mass ejection-driven shocks in solar fast-wind regions. Astrophys. J., 584, 1063-1070, doi: 10.1086/345780. [Link]

Kasatkina, E. A., O. I. Shumilov, M. J. Rycroft, F. Marcz, and A. V. Frank-Kamenetsky, 2009: Atmospheric electric field anomalies associated with solar flare/coronal mass ejection events and solar energetic charged particle "Ground Level Events". Atmos. Chem. Phys. Discuss., 9, 21941-21958.

Kumar, A. and H. P. Singh, 2013: Impact of high energy cosmic rays on global atmospheric electrical parameters over different orographically important places of India. ISRN High Energy Physics, 2013, 1-7, doi: 10.1155/2013/831431. [Link]

Lay, E. H., R. H. Holzworth, C. J. Rodger, J. N. Thomas, O. Pinto Jr., and R. L. Dowden, 2004: WWLL global lightning detection system: Regional validation study in Brazil. Geophys. Res. Lett., 31, L03102, doi: 10.1029/2003GL018882. [Link]

Li, G., G. P. Zank, and W. K. M. Rice, 2003: Energetic particle acceleration and transport at coronal mass ejection-driven shocks. J. Geophys. Res., 108, doi: 10.1029/2002JA009666. [Link]

Martin, R., 2009: Relations between solar activity and solar tides caused by the planets defined. Available at http:// climatestop.com/SolarTidalInfluencesofVEJ W01.pdf.

Masuda, S., T. Kosugi, H. Hara, S. Tsuneta, and Y. Ogawara, 1994: A loop-top hard X-ray source in a compact solar flare as evidence for magnetic reconnection. Nature, 371, 495-497, doi: 10.1038/371495a0. [Link]

Molinari, J., P. Moore, and V. Idone, 1999: Convective structure of hurricanes as revealed by lightning locations. Mon. Weather Rev., 127, 520-534, doi: 10.117 5/1520-0493(1999)127<0520:CSOHAR>2.0.CO;2. [Link]

Murphy, N. A., M. P. Miralles, C. L. Pope, J. C. Raymond, H. D. Winter, K. K. Reeves, D. B. Seaton, A. A. van Ballegooijen, and J. Lin, 2012: Asymmetric magnetic reconnection in solar flare and coronal mass ejection current sheets. Astrophys. J., 751, 1-13, doi: 10.1088/0004-637X/751/1/56. [Link]

Raymond, J. C., A. Ciaravella, D. Dobrzycka, L. Strachan, Y. K. Ko, M. Uzzo, and N. E. Raouafi, 2003: Far-Ultraviolet spectra of fast coronal mass ejections associated with X-class flares. Astrophys. J., 597, 1106-1117, doi: 10.1086/378663. [Link]

Rodger, C. J., S. Werner, J. B. Brundell, E. H. Lay, N. R. Thomson, R. H. Holzworth, and R. L. Dowden, 2006: Detection efficiency of the VLF World-Wide Lightning Location Network (WWLLN): Initial case study. Ann. Geophys., 24, 3197-3214, doi: 10.5194/angeo-24-3197-2006. [Link]

Sentman, D. D., 1995: Schumann resonances. In: Volland,
H. (Ed.), Handbook of Atmospheric Electrodynamics I, CRC Press, Boca Raton, Fla, 267-298.

Shibata, K., 1996: New observational facts about solar flares from Yohkoh studies - Evidence of magnetic reconnection and a unified model of flares. Adv. Space Res., 17, 9-18, doi: 10.1016/0273-1177(95)00534-L. [Link]

Sorokin, V. M., A. K. Yaschenko, V. M. Chmyrev, and M. Hayakawa, 2006: DC electric field formation in the mid-latitude ionosphere over typhoon and earthquake regions. Phys. Chem. Earth, 31, 454-461, doi: 10.1016/j.pce.2005.09.001. [Link]

Tonev, P. T. and P. I. Y. Velinov, 1996: A quasi-DC model of electric fields in the ionosphere-ground region due to electrified clouds. J. Atmos. Terr. Phys., 58, 11171124, doi: 10.1016/0021-9169(95)00112-3. [Link]

Webb, D. F., 2000: Understanding CMEs and their source regions. J.Atmos. Sol.-Terr. Phys., 62, 1415-1426, doi: 10.1016/S1364-6826(00)00075-4. [Link]

Williams, E. R., 1994: Global circuit response to seasonal variations in global surface air temperature. Mon. Weather Rev., 122, 1917-1929, doi: 10.1175/1520-049 3(1994)122<1917:GCRTSV>2.0.CO;2. [Link]

\section{APPENDIX: GLOSSARY}

Venus transit: Passage of Venus over the surface of the Sun. During this passage Earth, Venus and Sun come into the same line.

Solar flares: Sudden brightening of the small area of the photosphere that may last between a few minutes and several hours.

Coronal Mass Ejections (CMEs): It is a continuous transient solar event occurring from the entire coronal zone of the Sun when bursty solar activity launches solar masses, e.g., protons, electrons, neutrons and also solar wind in the form of plasma into the heliosphere. It belongs to the most violent and fascinating phenomena in the solar system.

X-ray/ $\gamma$-ray burst: During magnetic reconnection of dipole magnetic field lines with the interplanetary field, huge energy is evolved as electromagnetic radiation that releases the trapped masses along with the electromagnetic radiation in the form of X-ray $/ \gamma$-ray burst.

Ionization of the D-region of the ionosphere: Ionization of the constituent particles of the D-region of the ionosphere by the intense radiation coming from Sun.

Modification of Earth-ionosphere waveguide: Dielectric and magnetic properties of the medium within Earth-ionosphere waveguide covering D-region of the ionosphere will be modified.

Atmospheric electricity: Electricity generated by natural processes like solar radiation, lightning and various geomagnetic processes in the Earth's atmosphere which have very high impact in different atmospheric processes. Parameters like SR, PDC, VLF, ELF, PG from ground surface are 
very much responsible to control the meteorological connections and some other phenomena related to atmospheric electricity.

Schumann resonance (SR): Within the spherical cavity between the Earth's surface and lower ionosphere, standing electromagnetic waves are generated due to excitation energy from lightning. The spherical cavity acts as a resonator. The resonance occurs when the wavelength is of the order of the resonator dimension. A subset of such resonances is the SR. The observed discrete peak frequencies $8,14,20,26, \ldots \mathrm{Hz}$ in the extremely low frequency (ELF) range are generated by lightning discharges.

ELF: Extremely low frequency (ELF) electromagnetic radiation in the frequency range $3-300 \mathrm{~Hz}$.

VLF: Very low frequency (VLF) electromagnetic radiation in the frequency range $3-30 \mathrm{kHz}$.

FWF: Fair weather is an atmosphere of sunny weather without any precipitation, without any appreciable amount of low clouds, without the occurrence of any sort of charge transfer process, with calm or with low winds. The atmospheric electric field during such situation is FWF. Field strength near the Earth's surface is $\sim 130 \mathrm{Vm}^{-1}$. Carnegie's oceanic field curve is widely accepted FWF curve.

PG: Global weather activity maintains a potential difference of about $250 \mathrm{kV}$ between Earth-ionosphere waveguide which generates a potential difference between the ground surface and any point vertically above the surface of the Earth. This difference is called potential gradient and its value measured from Kolkata is about $120 \mathrm{Vm}^{-1}$.

PDC: The existence of an electric field in the atmosphere causes an ionic current to flow from atmosphere to Earth or vice versa through sharp points. This is known as Point Discharge Current. The direction of the current is governed by the direction of the field.

Dst Index: It is a measure of the variations in the geomagnetic field due to the equatorial ring current. At a given time, the Dst index is the average of the variations in the H-component over all longitudes. The reference level is selected such that Dst is statistically zero on internationally designated quiet days. An index smaller than $-50 \mathrm{nT}$ indicates the absence of magnetic disturbance.

Kp Index: It is measurement of disturbances in the horizontal component of Earth's magnetic field ranging between 0 - 9. It is derived from the maximum fluctuations of horizontal components of the Earth's magnetic field observed on a magnetometer during a three-hour interval. The values of this index give a good indication of the geomagnetic activity, whereby the value 1 being calm and 5 or more indicate a geomagnetic storm. Values between 0 - 4 indicate quiet magnetic conditions. 